\title{
Comunicación
}

\section{Brote de jacapo en ovinos en Junín, Perú}

\author{
AN OUTBREAK OF PHOTOSENSITIZATION (JACAPO) IN SHEEP \\ in Junin, Peru
}

Katherine Choez A. ${ }^{1,2}$, Pol Galarza Z. ${ }^{1}$

\section{Resumen}

Se describe un brote de jacapo en el distrito de Carhuamayo, departamento de Junin, Perú, que afectó a 10 animales de un rebaño de 150 ovinos Corriedale, criados bajo un sistema de crianza extensiva. Los animales afectados presentaron lesiones oculares y cutáneas en la región de la cabeza con diferentes grados de severidad. El jacapo fue descrito hace más de 20 años como una enfermedad esporádica en Perú; sin embargo, por efectos del cambio climático y el deterioro de la capa de ozono se está convirtiendo en una enfermedad emergente que viene afectando a la ganadería ovina de la sierra central del país.

Palabras clave: jacapo; fotosensibilización; ovinos; Junín

\section{Abstract}

An outbreak of photosensitization (locally called jacapo) in the district of Carhuamayo, department of Junin, Peru, that affected 10 animals of a flock of 150 Corriedale sheep is described. The flock was reared under an extensive breeding system. The affected animals presented ocular and cutaneous lesions in the region of the head with different degrees of severity. The jacapo was described more than 20 years ago as a sporadic disease in Peru; however, because of climate change and the deterioration of the ozone layer, it is becoming an emerging disease that is affecting sheep flocks in the central highlands of the country.

Key words: jacapo; photosensitization; sheep; Junín

\footnotetext{
${ }^{1}$ Facultad de Medicina Veterinaria y Zootecnia, Universidad Peruana Cayetano Heredia, Lima, Perú

${ }^{2}$ E-mail: kathyvet3@hotmail.com
}

Recibido: 29 de enero de 2018

Aceptado para publicación: 30 de junio de 2018 


\section{INTRODUCCIÓN}

Jacapo es el término con el que se le conoce en el Perú a la fotosensibilización del ganado ovino. La fotosensibilización es una reacción hiperactiva de la piel frente a la luz ultravioleta, debida a la presencia de sustancias fototóxicas o fotoactivas en la misma (Puschner et al., 2016), que ocasiona dermatitis, hiporexia, pérdida de peso y, en algunos casos, la muerte de los animales afectados (Radostitis et al., 2007). El jacapo no se presenta cuando la piel está pigmentada, protegida por una amplia capa de lana o pelo o si los animales permanecen en la sombra (Mulero, 2004).

Existen tres tipos de fotosensibilización: Tipo I (primaria), Tipo II (congénita) y Tipo III (hepatógena). La manifestación primaria surge debido a la ingestión de sustancias fotoactivas exógenas contenidas en las plantas (Haargis y Ginn, 2007; Radostitis et al., 2007). La fotosensibilización congénita es rara en animales domésticos, y está asociada con el metabolismo aberrante de las porfirinas, lo que resulta en la acumulación de sustancias fotoactivas dentro de la sangre y los tejidos. La fotosensibilización hepatógena se produce cuando se ve afectada la capacidad del hígado para excretar la filoeritrina, derivada del catabolismo de la clorofila alimentaria (Haargis y Ginn, 2007), de modo que se acumula llegando a los capilares dérmicos, permitiendo que la luz ultravioleta cause lesiones en la piel (Smith, 2000; Scheie et al., 2002).

La fotosensibilización se ha descrito en diferentes especies animales (Witte y Curry, 1993; Wisløff et al., 2002; Garoussi, 2006; Rossanigo et al., 2010; De Oliveira et al., 2013; Puchner et al., 2016). En la sierra central del Perú se han reportado casos con una incidencia de 0.18 y $0.10 \%$ en la SAIS Túpac Amaru y la SAIS Cahuide, respectivamente, debida a la ingestión de especies de plantas del género Astragalus (garbancillo) (Espinoza et al., 1989). Ha sido descrita como una enfermedad estacional y esporádica, que ocurre especialmente en las épocas del llamado «verano» en las serranías del país, esto es entre mayo y agosto, cuando los rayos solares son fuertes durante el día y la noche es fría por las fuertes heladas (Espinoza et al., 1989).

\section{Caso Clínico}

El presente brote se presentó en noviembre de 2017, en un fundo ubicado en el distrito de Carhuamayo, departamento de Junin, Perú, ubicado a $4100 \mathrm{msnm}$. El fundo contaba con un rebaño de 150 ovinos Corriedale bajo un sistema de crianza extensiva y alimentación a libre pastoreo, sin suplementos. Las pasturas predominantes en la zona eran de los géneros Festuca, Calamagrostis, Stipa y Trifolium.

Se encontraban afectados 10 animales, entre ovejas, carneros y corderos. Los animales presentaron signos de fotosensibilización en la región de la cabeza; ya que esta zona no se encuentra pigmentada y está desprovista de lana y, por lo tanto, más expuesta a los rayos ultravioleta. A la inspección se pudo observar lesiones cutáneas en los 10 animales con diferentes grados de severidad, caracterizadas por congestión y eritema en la cara, que con el paso de los días se edematizaban y formaban pequeñas vesículas que estallaban, liberando una secreción serosa que resultaba en costras. En algunos animales se pudo observar, además, edema de cara, labios, orejas, párpados y zona nasal. Además, se encontraron problemas oculares como edema palpebral, congestión y edema de conjuntivas, epifora y fotofobia. Así mismo, los animales estaban en la búsqueda de sombra y se frotaban constantemente las zonas afectadas debido al intenso prurito.

Al examen clínico, las ovejas estaban deprimidas y habían bajado su condición corporal, experimentando de una moderada a 

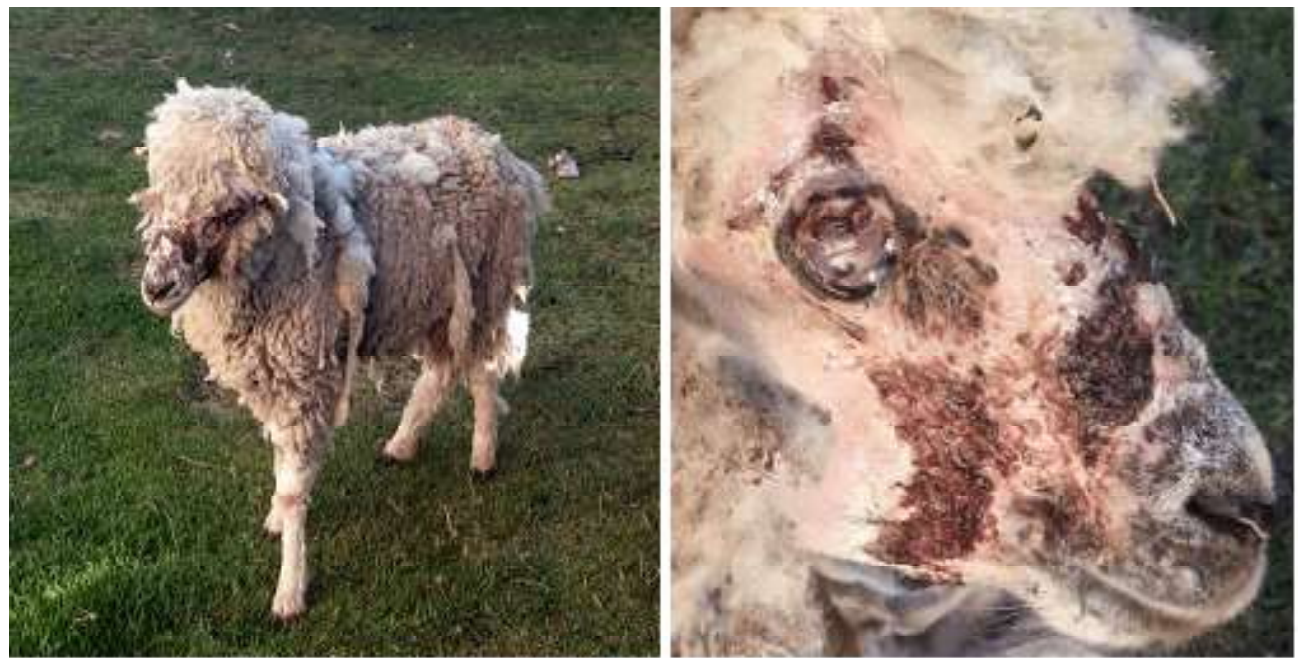

A

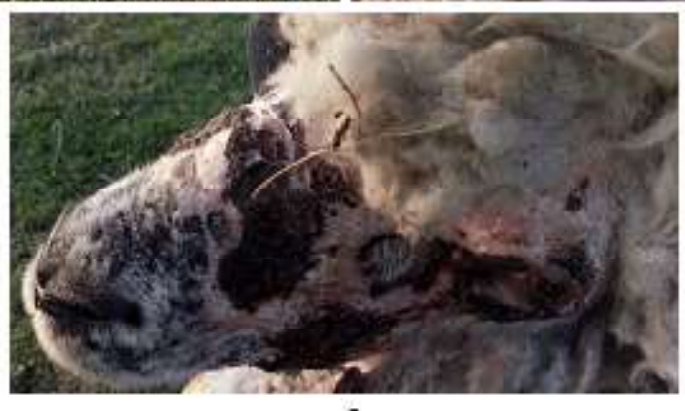

Figura 1. Oveja de dos años severamente afectada. A. Vista general del animal; B. Dermatitis severa con congestión y eritema en la cara, ulceración corneal grave. C. Zonas de necrosis con desprendimiento de la piel afectada

total anorexia, especialmente los animales que presentaron zonas edematosas a nivel de labios y papada. Las lesiones más graves se presentaron en una oveja de dos años, con lesión bilateral de los ojos, ulceración corneal grave y dermatitis severa, con secreciones mucopurulentas en algunas áreas y necrosis con desprendimiento de la piel afectada (Figura 1).

El tratamiento realizado fue sintomático. Los animales afectados fueron retirados del pastoreo y dejados en áreas sombreadas y con disposición de agua y alimento, aunque los animales menos afectados podían salir a pastar en las horas de menor exposición so- lar. Así mismo, se aplicó un antihistamínico a base de cloroprofenpiridamida por vía IM c/2 días para bloquear los efectos causados en el organismo por la liberación de histamina y un antibiótico a base de oxitetraciclina endovenoso por cinco días seguidos. Además, las heridas fueron limpiadas con antisépticos para evitar la complicación bacteriana.

A las cuatro semanas de aislar a los animales de la exposición solar, el eritema y la congestión desaparecieron; sin embargo, las lesiones más profundas tardaron más tiempo en sanar. La oveja severamente afectada se mantuvo con antibióticos y antisépticos por tres semanas, hasta que sus lesiones cicatri- 
zaron por segunda intención, dando lugar a costras que se desprendieron al cabo de tres meses; no obstante, quedó ciega debido a la severa lesión ocular.

\section{Discusión}

El jacapo fue reportado por primera vez en el Perú en 1989 (Espinoza et al., 1989), siendo descrita como una enfermedad esporádica con presentación entre los meses de mayo y agosto; sin embargo, por los posibles efectos del cambio climático y el deterioro de la capa de ozono, los rayos ultravioleta inciden con mayor potencia sobre la tierra (Amron y Moy, 1991), activando las sustancias fototóxicas presentes en la piel de los animales, ocasionando severas dermatitis (Radostitis et al., 2007). Estas lesiones ocasionan pérdidas económicas en el poblador altoandino por baja conversión alimenticia, pérdida de peso y muerte de los animales (Ochoa, 2012).

Las lesiones se observaban en todos los casos en la zona de la cabeza, la cual es despigmentada y carente de lana, lo cual coincide con otros reportes (Rossanigo et al., 2010; De Oliveira et al., 2013; Puschner et al., 2016). Se sabe que la piel pigmentada o la presencia de lana no permite que los rayos ultravioleta incidan fuertemente sobre la piel (Mulero, 2004), y de esta manera no se genera fotorreacción.

Este brote de jacapo se presentó en una época que no había sido reportado previamente (noviembre), pero coincidente con días de altas temperaturas y luminosidad en la zona. Podría considerarse a la fotosensibilización como una enfermedad emergente (Rossanigo et al., 2011) que afecta a varias especies animales en Perú, pero que lamentablemente no es reportado. Además, no se han realizado más investigaciones acerca de pasturas presentes en el territorio nacional que pudieran causar un tipo de fotosensibilización primaria, pues solo se dispone del trabajo de años atrás de Espinoza et al. (1989).

En el presente brote no se llegó a determinar el tipo de fotosensibilización que afectó a los animales, pero es un referente para futuras investigaciones, y sobre todo para tomar conciencia de la importancia del aumento de los niveles de los rayos ultravioleta en estos últimos años, con el propósito de implantar políticas de prevención.

\section{Literatura Citada}

1. Amron DM, Moy RL. 1991. Stratospheric ozone depletion and its relationship to skin cancer. J Dermatol Surg Onc 17: 370-372. doi: 10.1111/ j.1524-4725.1991.tb01713.x

2. de Oliveira CHS, Barbosa JD, Oliveira CMC, Bastianetto E, Melo MM, Haraguchi M, Freitas LGL, et al. 2013. Hepatic photosensitization in buffaloes intoxicated by Brachiaria decumbens in Minas Gerais state, Brazil. Toxicon 73: 121-129. doi: 10.1016/ j.toxicon.2013.07.001

3. Espinoza J, San Martín F, Holgado D, Suárez F. 1989. Intoxicación por plantas venenosas que afectan a la ganadería peruana. En: Investigaciones sobre pastos y forrajes de Texas Tech University en el Perú. Costa Rica: RISPAL. p 83-96.

4. Garoussi M. 2006. The frequency of photosensitization in a dairy cattle herd infected by Leptospires. Scientific report. Iran J Vet Res 7: 70-72. doi: 10.22099/ IJVR.2006.2686 
5. Haargis AM, Ginn PE. 2007. The integument. In: McGavin MM, Zachary JF (eds). Pathologic basis of veterinary disease. $4^{\text {th }}$ ed. St. Louis, USA: Elsevier. p 1107-1261.

6. Mulero AM. 2004. Efecto de la radiación ultravioleta (RUV) sobre los procesos de estrés oxidativo e inmudrepresivos cutánea. Efecto protector de los filtros solares. Tesis Doctoral. Cataluña, España: Univ. Rovira y Virgili. 178 p.

7. Ochoa OW. 2012. Toxicología de la fotosensibilización en ganaderías de leche. Tesis de Médico Veterinario Zootecnista. Cuenca, Ecuador: Univ. de Cuenca. $113 \mathrm{p}$.

8. Puschner B, Chen X, Read D, Affolter VK. 2016. Alfalfa hay induced primary photosensitization in horses. Vet $\mathrm{J} 211$ : 32-38. doi: 10.1016/j.tvj1.2016.03.004

9. Radostits OM, Gay CC, Hinchcliff KW, Constable PD. 2007. Veterinary medicine: a textbook of the diseases of cattle, horses, sheep, pigs and goats. $10^{\text {th }}$ ed. Edinburgh, UK: Saunders Elsevier. $521 \mathrm{p}$.

10. Roder JD. 2002. Patofisiología de la generación de radicales libres. En: Manual de toxicología veterinaria. Barcelona: Multimédica. p 57-60.

11. Rossanigo CE, Bengolea A, Sager RL. 2011. Patologías emergentes de la intensificación bovina en la región semiárida
- subhúmeda del centro de la Argentina. Mercedes, Argentina: INTA. 29 p.

12. Rossanigo CE, Toselli J, Miranda AO, Bengolea A. 2010. Casos de fotosensibilización y uveítis en tres especies en la región subhúmeda del centro de la Argentina por consumo de alfalfa (Medicago sativa). En: XVIII Reunión Científico Técnica de la Asociación Argentina de Veterinarios de Laboratorios de Diagnóstico (AAVLD). Mercedes, Argentina.

13. Scheie E, Flåoyen A, Moan J, Berg K. 2002. Phylloerythrin: mechanisms for cellular uptake and location, photosensitization and spectroscopic evaluation. New Zeal Vet J 50: 104-110. doi: 10.1080/00480169.2002.36291

14. Smith BL. 2000. Effects of low dose rates of sporidesmin given orally to sheep. New Zeal Vet J 48: 176-181. doi: 10.1080/00480169.2000.36189

15. Wisloff, H, Wilkins AL, Scheie E, Flåoyen A. 2002. Accumulation of sapogenin conjugates and histological changes in the liver and kidneys of lambs suffering from alveld, a hepatogenous photosensitization disease of sheep grazing Narthecium ossifragum. Vet Res Commun 26: 381-396. doi: 10.1023/ A:1016298929610

16. Witte ST, Curry SL. 1993. Hepatogenous photosensitization in cattle fed a grass hay. J Vet Diagn Invest 5: 133-136. $10.1177 / 104063879300500135$ 